\title{
AGO Recommendations for the Diagnosis and Treatment of Patients with Advanced and Metastatic Breast Cancer: Update 2016
}

\author{
Marc Thilla Cornelia Liedtke ${ }^{b}$ on behalf of the AGO Breast Committee* \\ ${ }^{a}$ Klinik für Gynäkologie und Geburtshilfe, Agaplesion Markus Krankenhaus, Frankfurt/M., Germany; \\ ${ }^{b}$ Klinik für Frauenheilkunde und Geburtshilfe, Universitätsklinikum Schleswig-Holstein/Campus Lübeck, Lübeck, Germany
}

\author{
Keywords \\ Guidelines, locoregional relapse, metastatic breast \\ cancer, targeted therapy
}

\section{Introduction}

For the last 15 years, the Breast Committee of the German Gynecological Oncology Group (Arbeitsgemeinschaft Gynäkologische Onkologie, AGO) has been preparing and updating evidence-based recommendations for the diagnosis and treatment of patients with early and metastatic breast cancer. The AGO Breast Committee consists of gynecological oncologists specialized in breast cancer and interdisciplinary members specialized in pathology, radiological diagnostics, medical oncology, and radiation oncology. This update has been performed according to a documented rule-fixed algorithm, by thoroughly reviewing and scoring chapter by chapter

${ }^{*}$ Members of the 'AGO Breast Committee' in alphabetical order: U.-S. Albert, Frankfurt/M I. Bauerfeind, Landshut; J. Bischoff, Dessau; J.-U. Blohmer, Berlin; W. Budach, Düsseldorf; P. Dall, Lüneburg: I.J. Diel, Mannheim; T. Fehm, Düsseldorf; N. Fersis, Bayreuth; M. Friedrich, Krefeld; B. Gerber, Rostock; V. Hanf, Fürth; N. Harbeck, Munich; J. Huober, Ulm; C. Jackisch, Offenbach; W. Janni, Ulm; H. Kreipe, Hanover; T. Kühn, Esslingen; S. Kümmel, Essen; C. Liedtke, Lübeck; S. Loibl, Neu-Isenburg; H.-J. Lück, Hanover; M. Lux, Erlangen; N. Maass, Kiel; G. von Minckwitz, Neu-Isenburg; V. Möbus, Frankfurt/M.; V Müller, Hamburg; C. Mundhenke, Kiel; U. Nitz, Mönchengladbach;Dr. M. Rezai, Düsseldorf; A. Rody, Lübeck; A. Scharl, Amberg; M. Schmidt, Mainz; R. Schmutzler, Cologne; A Schneeweiss, Heidelberg; I. Schreer, Hamburg; F. Schütz, Heidelberg; P. Sinn, Heidelberg, E. Solomayer, Homburg; E. Stickeler, Aachen; M. Thill, Frankfurt/M.; C. Thomssen, Hal le/Saale; M. Untch, Berlin; F. Wenz, Mannheim; I. Witzel, Hamburg. Associated Members of the DEGRO: P. Feyer, Berlin; D. Rades, Lübeck.

The members of the AGO Breast Committee are also listed in the appendix accessible at www.karger.com/?DOI=447030 the recent publications for their scientific validity (Oxford level of evidence (LoE), www.cebm.net) [1] and clinical relevance (AGO grades of recommendation (GR); table 1). We present the 2016 update; the full version of the updated slide set is available online as a PDF file in both English and German [2]. Moreover, a version for patients is also available at www.ago-online.de.

\section{Bone Health and Osteo-Oncology}

\section{Bone Health}

Endocrine treatment with aromatase inhibitors (AIs) is associated with significant bone loss in most of postmenopausal women. Therefore, preventive measures should be undertaken including exercise, vitamin D (800-2000 U/day), reducing alcohol, cessation of smoking and avoiding a body mass index of less than $20 \mathrm{~kg} / \mathrm{m}^{2}$. In addition to lifestyle interventions, bisphosphonates (BPs) and denosumab can be offered for prevention and therapy of therapy-induced bone loss. Based on a recent meta-analysis by Coleman et al. [3], adjuvant BPs are associated with reduced breast cancer mortality and recurrence in postmenopausal women. Therefore, BPs including clodronate and aminobisphosphonates may be offered to improve clinical outcome in postmenopausal patients (LoE 1a/A/ AGO +). For denosumab, first survival data of the ABCSG-18 trial involving more than 3,400 postmenopausal patients were presented at the SABCS 2015 [4]. The primary study aim of the ABCSG-18 trial was to measure the time from randomization to first clinical fracture in those receiving denosumab versus those receiving the placebo. Compared with the placebo group, patients in the denosumab group had a significantly delayed time to first clinical fracture (hazard ratio (HR) 0.50, 95\% confidence interval (CI) 0.39$0.65), p<0.0001)$. Secondary endpoint of this study was diseaserelated outcome. At a median follow-up of 4 years, denosumab was

\section{KARGER}

Fax +497614520714
() 2016 S. Karger GmbH, Freiburg

$1661-3791 / 16 / 0113-0216 \$ 39.50 / 0$
PD Dr. Marc Thill

Clinic for Gynecology and Obstetrics. Interdisciplinary Breast Center

Agaplesion Markus Hospital, Frankfurt/Main

Wilhelm-Epstein-Straße 4, 60341 Frankfurt/M., Germany

marc.thill@fdk.info 
Table 1. AGO grades of recommendation

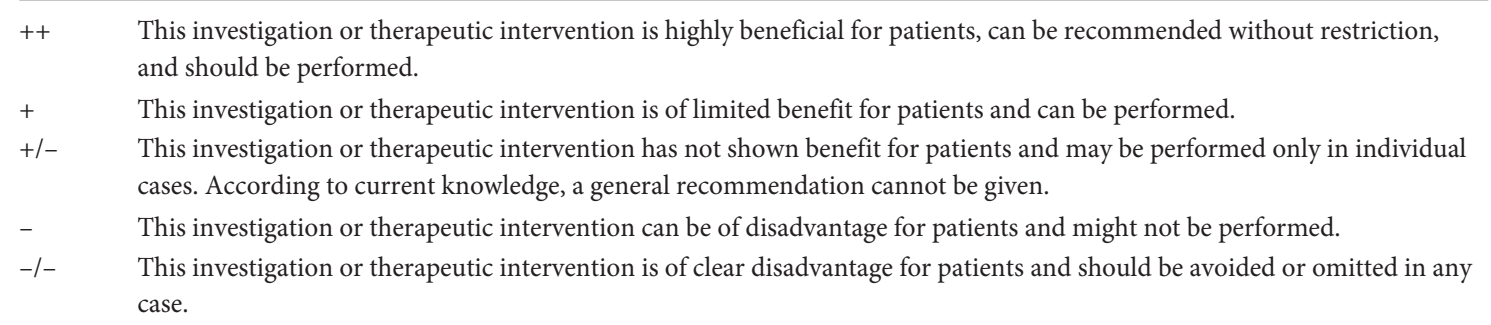

associated with a trend towards improved disease-free survival (DFS) (167 vs. 203 DFS events, HR 0.82, p = 0.051). Overall survival data are pending. The results of the D-CARE study examining denosumab as adjuvant treatment (NCT01077154) are also awaited to provide more information on survival benefit. Therefore, based on current data, BPs are still the preferable osteo-oncological adjuvant treatment for improving clinical outcome until more data are available for denosumab (LoE $\left.1 b^{\mathrm{a}} / \mathrm{B} / \mathrm{AGO}+/-\right)$.

\section{Bone Metastasis}

Osteoclast-inhibiting therapy including denosumab and BPs to reduce the risk of skeletal complications is the standard treatment for breast cancer patients with bone metastases. The approved schedule of administration for zoledronic acid (4 mg intravenously) and denosumab (120 mg subcutaneously) is every 3-4 weeks. Given concerns regarding the toxicity of these agents, including osteonecrosis of the jaw, there has been increasing interest in de-escalation of osteoclast inhibitors.

Over the past few years, several randomized phase III trials have compared treatment with zoledronate every 4 weeks to treatment every 12 week after completion of several months of treatment with an every 4-week schedule (ZOOM trial, OPTIMIZE-2 trial) or at initiation of treatment (CALGB (ALLIANCE) 70604) [5-7]. No significant differences in the rate of skeletal-related events (SREs) (e.g. pain, radiotherapy or fractures) from these 3 trials were reported.

The safety of less frequent dosing for osteoclast inhibitors is further supported by a meta-analysis of Ibrahim et al. [8] that included 5 clinical trials. The summary risk ratio for on-study SREs in patients receiving standard (61 of 443 patients) versus less frequent dosing (49 of 392 patients) therapy was 0.90 (95\% CI $0.63-1.29)$.

Therefore, the switch from a 4 -week to a 12 -week schedule after 1 year of monthly zoledronate may be an important option for metastatic breast cancer patients to reduce toxicities (LoE 1a/A/ AGO +). Clinical data addressing less frequent dosing than every 4 weeks for denosumab derive only from small phase II trials [9, 10]. Therefore, a de-escalation approach for denosumab is currently not recommended (LoE 4/C/AGO -).

\section{Follow-Up of Breast Cancer}

One of the major goals of breast cancer follow-up is the early detection of curable breast cancer events, i.e. breast or locoregional recurrence. The early detection of symptomatic metastases is desirable, but in terms of the early detection of asymptomatic metastases, data are inconsistent and most importantly do not suggest a survival benefit. Beyond improvement of survival, additional issues like improvement of quality of life, physical performance and the reduction of treatment-related side effects are important (LoE2b/B/ AGO +). In addition, re-evaluation of current adjuvant therapies and the assessment or improvement of treatment compliance (especially endocrine therapy) should be part of follow-up (LoE5/D/ AGO ++). It should thus be pointed out that every patient has the right to obtain a second opinion $(\mathrm{LoE} 2 \mathrm{c} / \mathrm{B} / \mathrm{AGO}++)$ as well as to undergo genetic counselling, hormone replacement, prophylactic surgery and breast reconstruction (LoE2c/C/AGO +). Further issues such as, for example, pregnancy, contraception, sexuality, quality of life, menopausal symptoms, and specific psychological aspects should be addressed proactively (LoE4/C/AGO +).

Life-style modifications (cessation of smoking, diet, reduced alcohol consumption and physical activity) and interventions with regard to co-morbidities (diabetes) are further important aspects of follow-up. From a patient's perspective, examination of the breast, reassurance, guidance with answering questions, evaluation of treatment with side effects and psychosocial support are also important.

Most importantly, follow-up examinations of asymptomatic patients in routine situations should not contain tumor-marker measurements, liver ultrasound, bone scans, X-ray, CT or PET scans and monitoring of circulating tumor cells (CTCs). Imaging to detect curable events (mammography, ultrasound and in specific situations MRI) in combination with self-examination and physical examination is recommended. In this context, screening for second malignancies according to guidelines (e.g. colorectal, endometrial, ovarian, cervical cancer and lymphoma) is meaningful.

Further Dexa Scan at baseline and repeat scan according to individual risk in women with premature menopause or women taking an AI is recommended [11]. See supplemental fig. 1: Followup care for breast cancer; $w w w$. karger.com $/$ ?DOI $=447030$.

\section{Locoregional Recurrence}

In patients with locoregional relapse, pretherapeutic biopsy to re-assess histology as well as estrogen receptor (ER), progesterone receptor (PR), and human epidermal growth factor receptor 2 
(HER2) status is strongly recommended (LOE $3 \mathrm{~b} / \mathrm{B} / \mathrm{AGO}++$ ) [12] Besides known clinico-pathological factors, obesity is also associated with poor prognosis and breast cancer-specific survival, regardless of menopausal status and starting time of obesity. This was shown in a recent meta-analysis of 82 studies $(n=213,075)$ (LOE 1b) [13]. In addition, high-risk patients according to multigene assays are also at increased risk for locoregional relapse [14, 15]. Clinical consequences, however, of these initial reports remain to be determined.

With regard to surgical options, a sentinel lymph node dissection (SLND) after prior SLN biopsy may be feasible in case of cN0 status (LOE $1 \mathrm{~b} / \mathrm{B} / \mathrm{AGO}+/-$ ). Axillary dissection should not be performed in cases with $\mathrm{cN} 0$ disease and lack of SLN detection. In a recent Danish case series, SLND was possible in 72/144 patients (50\%). The detection rate after prior SLND (66\%) was significantly higher than after prior axillary dissection (34\%) [16].

With regard to systemic therapy, the CALOR trial ( $\mathrm{n}=163$; median follow-up 4.9 years) demonstrated a significant benefit of postoperative chemotherapy in patients with fully excised locoregional relapse particularly in cases with ER-negative disease (LOE 2b/B/AGO +). The 5-year DFS rates were 69\% (95\% CI 56-79) and $57 \%$ [44-67] with and without chemotherapy, respectively (HR 0.59 ; $95 \%$ CI $0.35-0.99 ; \mathrm{p}=0.046$ ) [17]. If chemotherapy is planned, a preoperative approach may be considered. In cases with HER2-positive disease, chemotherapy in combination with HER2targeted therapy is an option (LOE 5/D/AGO +). It needs to be emphasized that patients with inoperable locoregional relapse were included in the pertuzumab registration trial CLEOPATRA, and thus the study results may also be relevant for this patient cohort. In patients with ER-positive locoregional relapse following complete resection (R0) endocrine therapy is considered standard $(\mathrm{LOE} 2 \mathrm{~b} / \mathrm{B} / \mathrm{AGO}++)$.

\section{Endocrine and Targeted Therapy in Metastatic Breast Cancer}

Endocrine therapy is the backbone of and first choice for the treatment of hormone receptor (HR)-positive metastatic breast cancer. To define the receptor status of metastatic breast cancer reliably at diagnosis, a biopsy and immunohistochemistry should be performed to determine the status of the metastatic site HR whenever possible. Pooled relative discordance rates between primary tumors and metastatic disease for ER, PR, and HER2 status have been reported in 20\% (95\% CI 16-35\%), 33\% (95\% CI 29-38\%), and $8 \%(95 \%$ CI $6-10 \%)$ of cases, respectively [18]. However, even if analyses showed persisting HR positivity, tumor cells may have generated resistance against endocrine treatment. Within all lines of treatment, treatment options should take previous endocrine therapies, age and comorbidities and drug approval status into consideration.

In peri- and premenopausal patients, the induction of ovarian function suppression (gonadotropin-releasing hormone (GnRH) analogue or luteinizing hormone analogue) is the first step in en- docrine treatment. Whenever possible, it should be combined with tamoxifen $20 \mathrm{mg} /$ day [19]. GnRH analogues can also be combined with an AI or with fulvestrant if there are contraindications for tamoxifen. The addition of the new CDK4/6 inhibitor palbociclib to the combination of $\mathrm{GnRH}$ analogue plus fulvestrant improved progression-free survival (PFS) significantly [20]. However, monotherapy with ovarian function suppression or tamoxifen alone remains an option but is not commonly considered standard of care.

In postmenopausal patients, endocrine treatment with fulvestrant $500 \mathrm{mg}$, an AI (crossed steroidal or non-steroidal AI depending on previous AI exposure) or tamoxifen are highly recommended [21, 22]. The phase II Paloma 1 trial showed a significant PFS advantage when letrozole and palbociclib were combined in the first-line setting [23] and led to provisional Federal Drug Administration (FDA) approval. A confirmatory phase III trial is currently ongoing. If the cancer progresses on therapy or shortly after single agent endocrine therapy, the combination of palbociclib with fulvestrant [24] or the combination of exemestane plus everolimus [25] could be considered. In the case of disease progression on 1 of the named agents at a later stage, an agent such as megestrole acetate or estradiol valerate (2-6 mg) may be used or previous treatments could be repeated. Initiation of first-line bevacizumab in parallel to endocrine therapy in the case of disease progression is not recommended ( $\mathrm{LoE} 1 \mathrm{~b} / \mathrm{B} / \mathrm{AGO}$-) (see supplemental fig. 2 ; www.karger.com/?DOI=447030).

For patients with remission or at least stable disease after firstline chemotherapy in combination with bevacizumab, maintenance therapy with bevacizumab combination with second-line endocrine therapy is recommended until disease progression or unacceptable toxicity (LoE 2b/B/AGO +) [26].

In HER2-positive/HR-positive advanced breast cancer, combinations of anastrozole or letrozole with trastuzumab, or letrozole with lapatinib, or fulvestrant with lapatinib are therapeutic options $[27,28]$. However, PFS was quite short in these clinical trials. Combination therapies of induction chemotherapy plus HER2-directed treatment should be considered a more effective option.

\section{Chemotherapy with or without Targeted Drugs in Metastatic Breast Cancer}

Treatment strategies in metastatic breast cancer are based on tumor biology, with the choice of treatment depending on HR and HER2 status. Other parameters guiding treatment selection are: (1) possible combination with targeted agents; (2) previous treatments (and their toxicities); (3) aggressiveness of the disease and location of metastases; (4) biological age; (5) comorbidities (including organ dysfunction); and (6) patient preference and expectations. In HR-positive tumors, endocrine therapy is generally preferred. If the leading site of metastases has been proven to be HR negative (preferentially diagnosed by biopsy of at least 1 metastatic lesion) or if the course of the disease suggests endocrine resistance or urgent need for response, cytotoxic chemotherapy is indicated (LoE 
1a/A/AGO ++). This is in accordance with the recently published Second International Consensus Guidelines for Advanced Breast Cancer (ABC 2) [29]. Monotherapy is preferred to polychemotherapy in non-life-threatening situations, as combination therapy increases response rates but not overall survival (OS) [30]. It is recommended to treat as long as the therapeutic index remains positive $(\operatorname{LoE} 1 \mathrm{a} / \mathrm{A} / \mathrm{AGO}++)$. Assuming a positive therapeutic index, the preferred option is to treat until progression ( $\mathrm{LoE} 2 \mathrm{~b} / \mathrm{B} / \mathrm{AGO}$ + ), compared with stopping treatment at best response (LoE $2 \mathrm{~b} / \mathrm{B} /$ AGO +/-). After adjuvant taxane and anthracycline treatment, a taxane re-challenge in first or later line therapy is possible and associated with a significant response that is dependent on treatment-free interval between progression and last taxane-containing chemotherapy (DFS < 1 year 34.8\%; DFS $1-2$ years $42.9 \%$ and DFS $>2$ years 63.3\%) ( $\mathrm{LoE} 2 \mathrm{~b} / \mathrm{B} / \mathrm{AGO}+)$ [31]. In case of comorbidities or severe side effects of prior treatment, metronomic chemotherapy may offer the possibility of prolonged treatment with fewer side effects ('high time, low dose'). Furthermore, as a maintenance treatment it can prolong the efficacy of conventional cytotoxic treatments (LoE 2b/B/AGO +) [32] (see supplemental fig. 3; www. karger.com/?DOI=447030).

A change of chemotherapy agents before tumor progression is recommended only as an exception ( $\mathrm{LoE} 2 \mathrm{~b} / \mathrm{B} / \mathrm{AGO}+/-)$, although 1 trial showed a benefit of capecitabine plus bevacizumab therapy compared to bevacizumab alone after a taxane/bevacizumab therapy [33]. Monitoring of treatment response should be performed by assessment of the tumor burden at baseline and approximately every 2-3 months, i.e. every 2-4 cycles of treatment. In slow-growing disease, longer intervals are reasonable $(\mathrm{AGO}++)$. The presence of CTCs determined with the standardized Cell Search ${ }^{\circledR}$ system (Janssen Diagnostics South Raritan, NJ, USA) is an adverse prognostic factor for outcome, and CTC dynamics are an early predictor of therapy response [34]. Given the unclear consequences, CTC determination is recommended preferentially within clinical trials $(\mathrm{LoE} 1 \mathrm{~b} / \mathrm{A} / \mathrm{AGO}+$ ) [35]. In some settings, e.g. in triple-negative breast cancer (TNBC) or aggressive tumor progression, combination of chemotherapy with bevacizumab may be used as this has been shown to improve response rates and PFS, albeit not survival $(\operatorname{LoE} 1 \mathrm{~b} / \mathrm{B} / \mathrm{AGO}+)$ [36-38]. Treatment with bevacizumab should usually be stopped in the case of disease progression, although 1 trial demonstrated a PFS benefit for the continuation with new lines of chemotherapy [39].

Platinum-based chemotherapy may be useful as treatment, especially in TNBC (LoE 1b/B/AGO +/-). Platinum compounds seem to be especially active in patients with BRCA mutations and, therefore, a higher level of recommendation in this group is justified $(\mathrm{LoE} 1 \mathrm{~b} / \mathrm{B} / \mathrm{AGO}+)[40]$. The most substantial progress in the past few years has been achieved in HER2-overexpressing disease. Pertuzumab in combination with trastuzumab and docetaxel has shown benefit regarding both PFS and OS as first-line therapy compared to docetaxel and trastuzumab alone [41], and is recommended as first-line therapy (LoE $1 \mathrm{~b} / \mathrm{A} / \mathrm{AGO}++$ ). A recently published study also showed activity and comparable efficacy for the combination of pertuzumab + trastuzumab + paclitaxel $(\operatorname{LoE} 2 \mathrm{~b} / \mathrm{B} /$
AGO +) [42]. Another first-line treatment option might be the combination of pertuzumab, trastuzumab and vinorelbine (LoE 3ba/B/AGO +/-) [43]. Furthermore, trastuzumab emtansine (TDM1) is approved as a further HER2-targeted treatment option in metastatic breast cancer. It has shown to enhance treatment efficacy (with respect to PFS and OS) in patients pretreated with trastuzumab either in comparison to lapatinib and capecitabine, or in heavily pretreated patients compared with treatment of physician's choice [44, 45]. Therefore, T-DM1 represents a recommended treatment option for patients with HER2-positive metastatic breast cancer in the first-line setting diagnosed $<6$ months after adjuvant therapy using taxane and trastuzumab ( $\mathrm{LoE} 1 \mathrm{~b} / \mathrm{B} / \mathrm{AGO}++)$, or at second or further lines of therapy after trastuzumab treatment $(\mathrm{LoE} 1 \mathrm{~b} / \mathrm{A} / \mathrm{AGO}++)$.

\section{Specific Sites of Metastases}

Specific sites of breast cancer metastases are liver, lung, pleura, pericardium, peritoneum, bone marrow, or any soft tissue. Other rare localizations such as adrenals, ovaries, uterus, stomach, colon, or placenta have also been reported; however, in such rare cases, controlled trials are not feasible, and treatment options must be discussed on an individual basis.

Management of primary stage IV breast cancer should focus primarily on systemic therapy, given that the impact of the extent of local treatment on patient survival is still a matter of debate. Although some trials suggested an association between local treatment (surgery or radiotherapy) of the primary tumor and prolonged survival, recent reports do not confirm these observations $[46,47]$. Therefore, controversy remains as to: (1) whether these results reflect a selection of women with good prognosis for primary site therapy; (2) what fraction of women in published studies were diagnosed with metastatic disease just after surgery, (3) whether specific subsets of metastases and biological subtypes would derive greater benefit, and (4) whether local therapy has been performed appropriately with regard to appropriate timing and extent. If surgery of the primary tumor is performed in the metastatic setting, local excision or mastectomy should be done with tumor-free margins $[48,49]$. Axillary surgery is only indicated for bulky disease. Local radiotherapy of primary tumor can be performed after local surgical treatment according to the indications of the adjuvant setting.

Systemic treatment of metastatic disease is the therapy of choice. Before treatment, metastases should be confirmed by histology to re-evaluate diagnosis of HR and HER2 status. Discordance regarding theses markers may occur in up to $45 \%$ of patients and may have impact on systemic treatment. If surgery for distant metastases is considered, good overall health, oligometastasis, and a long time between primary treatment and the occurrence of metastases are all favorable factors regarding outcome. Resection of liver metastases may be performed after histological verification if $\mathrm{R} 0$ resection is feasible, if no extrahepatic metastases are present, and tumor biology shows a HR-positive breast cancer responding 
well to former systemic therapy with a long disease-free interval and $\leq 3$ metastases $[50,51]$. In HER2-positive disease, the age should be $<50$ years and the metastasis smaller than $5 \mathrm{~cm}$. In these individual cases, 5-year-survival rates of $18-61 \%$ can be achieved $[52,53]$. Other procedures such as regional radiotherapy, stereotactic body radiosurgery with volumetric intensity-modulated arc therapy, thermoablation or chemoembolization are also possible in individual cases $[54,55]$.

For patients with pulmonary metastases, the LoE for a curative approach is low, but some patients might benefit from a metastasectomy followed by appropriate systemic treatment [56]. In accordance with the treatment of liver metastases, resection of lung metastases should only be performed with curative intent and if R0 resection is feasible and histological verification has been done (fine-needle aspiration with CT guidance or transbronchial needle aspiration). The timing of any local intervention may be critical; resection before progression is associated with a better outcome.

About $10 \%$ of all breast cancer patients develop malignant pleural effusion (MPE). In almost 50\% of MPE cases, it is the first sign of metastatic disease, resulting in dyspnea and reduced subjective well-being. It should be treated exclusively in symptomatic cases. Thoracoscopy with talcum pleurodesis or povidone-iodine $(20 \mathrm{ml}$ of $10 \%$ solution) (i.e. video-assisted thoracoscopy, VATS) is the option of choice for MPE. Other sclerosing but more rarely used agents are bleomycin, doxycycline, and mitoxantrone [57]. If expected life time is short, less invasive procedures should be considered. Continuous pleural drainage with indwelling pleural catheters is a well-tolerated and safe treatment alternative for patients who are not candidates for VATS. Catumaxomab is not yet recommended for MPE.

Overall, $3 \%$ of breast cancer patients will suffer from malignant ascites. Management of ascites takes place in the context of palliative care and aims at improving the quality of life of these patients. Patients with symptomatic ascites should undergo drainage. Local antibody therapy with catumaxomab remains an option in individual cases [58].

Malignant pericardial effusion and cardiac tamponade remain rare metastatic locations in patients with breast cancer. In symptomatic patients, drainage and pericardial fenestration are probably the treatment options of choice. For individual patients, VATS or ultrasound-guided puncture with instillation of mitoxantrone or bleomycin may be an alternative [59]. The choice between supportive care or specific anticancer treatment for poor performance status breast cancer patients with multi-metastatic disease and pancytopenia due to bone marrow involvement often remains a clinical and human dilemma. Depending on the underlying cancer biology, endocrine therapy or chemotherapy or antibody treatment options should be reconsidered [60]. It has been reported that aggressive combination treatment regimens were effective, since most patients showed improved marrow function after chemotherapy, and prolonged survival could be possible. Again, no significant changes to the recommendations in this chapter have been considered necessary.

\section{Central Nervous System Metastases in Breast Cancer}

Metastases to the CNS in breast cancer is of increasing clinical relevance since the development of brain metastases (BMs) has become a major limitation of life expectancy and quality of life for many patients. In patients with metastatic breast cancer, the incidence has increased to more than $30 \%$ in high-risk groups such as HER2-positive breast cancer or TNBC [61]. Despite of this high incidence, evidence for breast cancer-specific treatment approaches is very limited. Therefore, the AGO breast group encourages centers to participate in the German registry for breast cancer patients with BMs [62]. First results show that the survival from time of diagnosis of BMs is around 1 year for HER2-positive cases but shorter in the other groups. For optimization of treatment of breast cancer patients with BMs, a diagnosis-specific graded prognostic assessment was developed that takes into account the Karnofsky performance score, the biological subtype, number of BMs, and age [63].

The treatment strategy for patients with limited (1-3, in some studies also up to 4) BMs is not completely clear. As for other therapeutic options, no breast cancer-specific studies exist. Local therapy is the treatment of choice. This can be performed either as stereotactic radiotherapy (radiosurgery or fractionated stereotactic radiotherapy) or as surgery. In general, outcome is not improved by surgery compared with radiotherapy. Indications for surgery could be histological verification, e.g. after a long recurrence-free interval, need for immediate decompression in case of life-threatening symptoms and tumor size not allowing stereotactic radiotherapy. After surgery, radiotherapy of the resection area is recommended. The integration of whole-brain radiotherapy (WBRT) in the treatment concept for patients with a limited number of BMs is less clear. While the local control is better by adding WBRT, overall survival is not improved. Since WBRT leads to impairment of cognitive function without improvement in survival [64], local radiotherapy alone as first treatment option should be discussed with patients.

For patients with multiple BM, the treatment of choice is still WBRT. With the new radiation techniques, conformal avoidance of the hippocampal region and preservation of memory can possibly be improved [65].

Systemic therapy in patients with BMs should be performed as for other metastatic sites. In patients with HER2-positive disease, HER2-directed therapy should be continued if remission of extracranial disease is achieved. This recommendation is based on retrospective observations [66] and on studies demonstrating the penetration of trastuzumab into existing BMs [67]. In the future, the development of new therapeutic options with better brain penetration is of high relevance. For HER2-positive disease, new tyrosine kinase inhibitors (e.g. ONT-380) are in development and for HER2-negative disease, new chemotherapeutic options might be developed [68]. 


\section{Specific Situations}

In general, AGO recommendations in this chapter have remained virtually unchanged except for slight adaptations, as no really practice-changing papers have been published for the specific situations in breast cancer. For young women with breast cancer, a bi-annual international consensus meeting now exists summarizing recent management guidelines specific for these patients [69]. A recent meta-analysis (12 randomized controlled trials, $n=1,231$ ) found that ovarian suppression with $\mathrm{GnRH}$ to prevent chemotherapy-induced premature ovarian failure (POF) seemed to reduce the risk of POF (odds ratio (OR) 0.36, 95\% CI 0.23-0.57; p < 0.001) and be associated with an increase in pregnancies. In studies reporting DFS, no adverse effect on outcome could be demonstrated [70]. As substantial heterogeneity exists between trials, the AGO recommendation is still to be cautious with $\mathrm{GnRH}$ therapy during (neo-)adjuvant chemotherapy (LOE 1b/B/AGO +/-).

For male breast cancer patients, breast-conserving surgery is a safe option next to mastectomy as demonstrated in a large cohort from the SEER database [71], and is thus recommended by the AGO (LOE 4/C/AGO +). In the SEER cohort, only $47 \%$ received radiotherapy after breast-conserving surgery. Genetic counselling should be offered to male breast cancer patients even without an affected female relative (LOE $2 \mathrm{~b} / \mathrm{B} / \mathrm{AGO}+$ ). For sarcomas of the breast, staging should include CT scans of chest and abdomen and a brain MRI for angiosarcomas (LOE 4/D/AGO ++). Regional hyperthermia plus chemotherapy and/or radiotherapy can be used to improve local control, in particular in angiosarcomas (LOE 4/C/ $\mathrm{AGO}+$ ). For distant metastasis or unresectable tumors, antiangiogenic treatment can be offered in cases with angiosarcomas (LOE 4/C/AGO +) [72].

\section{Supportive Care}

The use of the granulocyte colony-stimulating factors (G-CSFs), filgrastim and pegfilgrastim, where indicated, is well established. In a comparative effectiveness study, pegfilgrastim prophylaxis was associated with a reduced risk of neutropenia-related or all-cause hospitalization relative to filgrastim prophylaxis [73]. A recent study in high-risk breast cancer demonstrated that $6 \mathrm{mg}$ lipegfilgrastim, a novel glyco-pegylated G-CSF, was as effective as pegfilgrastim in reducing neutropenia in patients with breast cancer receiving myelo-suppressive chemotherapy (LoE 1b/B/AGO +) [74]. Regarding prophylaxis of delayed chemotherapy-induced emesis, dexamethasone was not superior to aprepitant but instead had similar efficacy and toxicity in preventing delayed emesis in breast cancer patients treated with anthracycline plus cyclophosphamide chemotherapy and receiving the same antiemetic prophylaxis for acute emesis (LoE 1b/A/AGO ++) [75]. Finally, it was acknowledged and confirmed that combined standard oncological and palliative care should be considered early in the course of illness for patients with metastatic breast cancer and/or high symptom burden [76] (see supplemental fig. 4; www.karger.com/?DOI=447030).
Over the last decade, major concerns were raised regarding the use of erythropoiesis-stimulating agents (ESAs) in the treatment of chemotherapy-induced anemia (CIA). Inconsistent results were reported especially in the metastatic setting and by meta-analyses [77-79]. In contrast, no data were available for the adjuvant treatment of breast cancer patients. In the last few years, 3 major trials reporting results on the use of ESAs in the adjuvant setting have been published [80-82]. With the exception of the known elevated risk of thrombotic complications, these studies showed no negative impact of the use of ESAs on disease progression or mortality, indicating that ESAs are safe for the prevention and treatment of CIA in the adjuvant situation. However, these results are contradictory to guidelines in Canada and the USA and to the pharmaceutical manufacturers' product labels, which indicate that these agents should not be used when patients receive chemotherapy with curative intent.

\section{Therapy Side Effects}

Acute toxicity and (in most cases) 100-day mortality rates are well documented in the majority of phase III trials. Toxicities are graded according to World Health Organization or National Cancer Institute standards. Various cytotoxic anticancer drugs have their class-specific toxicity profiles. Anthracycline-based standard chemotherapy regimens in the adjuvant setting demonstrate relatively low acute toxicity, and treatment-related mortality rates are below $1 \%$. However, with respect to long-term side effects, cardiotoxicity is clinically relevant. In addition, the impact of the biological age on adjuvant decision-making has to be considered, e.g. by measuring the biological age using the comprehensive geriatric assessment (CGA) [83]. The risk of cardiotoxicity associated with trastuzumab has been reported to be $4 \%$ in monotherapy and $27 \%$ when administered in combination with anthracyclines and cyclophosphamide; however, life-threatening or severe adverse events are rare [84].

With respect to cardiac toxicity of treatment combinations, see the algorithm in supplemental figure 4,www.karger.com/? $D O I=447030$ (based on the Affinity/SAKK 2210 study protocol, SAKK = Schweizerische Arbeitsgemeinschaft für Klinische Krebsforschung, Swiss Oncology Research Network). The combination of pertuzumab and trastuzumab plus docetaxel did not increase the incidence of cardiac adverse events (CLEOPATRA study) [85].

The NeoALTTO study introduced lapatinib and trastuzumab in the neoadjuvant setting together with paclitaxel without major cardiac dysfunction, but a high incidence of diarrhea due to lapatinib was noted [86]. Several neuropsychological studies suggest an association between chemotherapy and long-lasting cognitive deficits, possibly related to therapy-induced structural and functional alterations in the brain [87].

Along with the integration of an increasing number of targeted agents into clinical routine an optimal management of side effects plays more and more an essential role in the medical treatment of breast cancer. 
Given the fact that non hematological toxicities such as hypertension (bevacizumab) $[88,89]$ and stomatitis or pneumonitis (everolimus) $[25,90]$ are in the focus of these new generations of anticancer drugs, an adequate knowledge of the class specific toxicity profiles should be mandatory for every oncologist working in this field of treatment.

First of all closely monitoring such patients is necessary, because early intervention is often indicated in case of drug related symptoms.

\section{Online Supplemental Material}

Supplemental fig. 1. Follow-up care for breast cancer.

Supplemental fig. 2. Treatment recommendations for postmenopausal patients with metastatic breast cancer after adjuvant AI.
Supplemental fig. 3. Cytotoxic therapy after adjuvant taxane and anthracycline treatment in HER2-negative/HR-positive breast cancer.

Supplemental fig. 4. Use of granulocyte colony-stimulating factors.

To access the supplemental figures, please refer to www.karger.com/? $D O I=447030$.

\section{Disclosure Statement}

The authors have no relevant affiliation or financial involvement with any organization or entity with financial interest in or financial conflict with the subject matter or materials discussed in the manuscript apart from the disclosed. M.T. has received consultant and speaker honoraria from AstraZeneca, Amgen, Boehringer-Ingelheim, Celgene, Genomic Health, GSK, Myriad, Novartis, Pfizer, pfm Medical, Pierre-Fabre, Serag-Wiessner, Surgiceye, Roche, Sysmex Europe and TEVA. C.L. has received consultant and speaker honoraria from Amgen, Celgene, Genomic Health, GSK, Nanostring, Novartis, Pfizer, PharmaMar, Pierre-Fabre, Roche, and TEVA.

\section{References}

References can be found in the appendix, please refer to www.karger.com/?DOI $=447030$. 